\title{
CÁC YẾU TỐ TÁC ĐộNG ĐẾN THÁI Độ CỦA NGƯờI TIÊU DÙNG ĐỐI VỚI QUẢNG CÁO QUA SMARTPHONE TẠI THÀNH PHỐ HỒ CHÍ MINH
}

\author{
FACTORS AFFECTING CONSUMERS' ATTITUDE TO SMARTPHONE \\ ADVERTISING IN HO CHI MINH CITY
}

\author{
Hà Nam Khánh Giao ${ }^{1}$, Đỗ thị Thùy Dung ${ }^{2}$
}

\begin{abstract}
Tóm tắt - Nghiên cứu đánh giá mức độ tác động của các yếu tố ảnh hương đến thái độ của người tiêu dùng đối với quảng cáo qua điện thoại thông minh (smartphone) tại Thành phố Hồ Chí Minh, bằng việc khảo sát 290 khách hàng. Phuoong pháp phân tích Cronbach's Alpha, phân tích EFA cùng với phân tích hồi quy bọi được sủ dụng với phuoong tiện SPSS. Kết quả cho thấy mức độ ảnh hưởng của các yếu tố đến thái độ của người tiêu dùng ở Thành phố Hồ Chi Minh đối với quảng cáo qua smartphone, theo tầm quan trọng giảm dần: tính giải trí, giá trị thông tin, độ tin cậy, sự không phiền nhiếu, sự cho phép và kiểm soát. Nghiên cứu đề ra một số hàm ý quản trị cho các nhà quảng cáo qua smartphone nhằm phuc vu khách hàng tốt hơn, đạt thành công trong kinh doanh.
\end{abstract}

Tù khóa: quảng cáo qua smartphone, tính giải trí, thông tin, độ tin cậy, sụ không phiền nhiễu, sụ cho phép và kiểm soát.

\footnotetext{
Abstract - This research is to examine factors affecting consumers' attitude to smartphone advertising in Ho Chi Minh City by questioning 290 consumers. The methodologies of Cronbach's Alpha, Exploratory Factor Analyzing (EFA) and linear multiple regressioning were used by SPSS program. The results show that there are five main factors affecting consumers' attitude to smartphone advertising in Ho Chi Minh City, ordered by the decreasing level of importance: entertainment, informativeness, credibility, non-

${ }^{1}$ Khoa Đào tạo Sau đại học - Trường Đại học Tài Chính - Marketing.

${ }^{2}$ Công ty $\mathrm{CP}$ TMDV Cổng Vàng.

Ngày nhận bài: 13/12/16, Ngày nhận kết quả bình duyệt: 06/02/17, Ngày chấp nhận đăng: 22/02/17
}

irritation, permission and control. From that, the research makes the suggestions to smartphone advertisers in order to improve customer service better.

Keywords: smartphone advertising, entertainment, informativeness, credibility, nonirritation, permission and control

\section{GIỚI THIÊ̂U}

Marketing qua điện thoại (mobile marketing) hiện không còn là khái niệm mới đối với thế giới nhưng tại Việt Nam, thị trường Mobile Marketing đang thực sự được biết đến và ứng dụng vào những năm gần đây. Tổng số thuê bao điện thoại được đăng ký và đang hoạt động trên toàn quốc năm 2015 là 148,5 triệu, trong đó smartphone chiếm 93,3 triệu và cả nước hiện có 121,12 triệu thuê bao phát sinh lưu lượng, trong đó thuê bao $3 \mathrm{G}$ đã chiếm $20 \%$ tổng thuê bao [1]. Điều này cho thấy, nhu cầu thị trường về smartphone tăng mạnh. Đây là cơ hội tốt cho các nhà marketing.

Hiệp hội Mobile Marketing [2] định nghĩa marketing trên thiết bị thông minh là việc sử dụng các công cụ không dây, thiết bị thông minh để truyền tải nội dung và tương tác với khách hàng. Người sử dụng smartphone đang là khách hàng mục tiêu của nhiều thương hiệu cao cấp. Việc sử dụng smartphone để truy cập internet, kiểm tra e-mail, tương tác với bạn bè trở nên phổ biến với người tiêu dùng Việt Nam. Do đó, quảng cáo qua smartphone được xem là kênh quảng cáo tiềm năng tại thị trường Việt Nam. Tuy nhiên, vẫn còn nhiều khách hàng có cảm giác bị phiền toái, thậm chí cảm nhận sự lừa đảo, không đáng tin cậy (quảng cáo qua SMS, tin nhắn rác, spam). Việc nghiên cứu về thái độ người tiêu 
dùng đối với quảng cáo qua smartphone trở nên rất cần thiết.

\section{CƠ SỞ LÝ LUẬN}

\section{A. Khái niệm quảng cáo qua smartphone}

Theo Kotler và Amstrong [3], tổ hợp chiêu thị có 5 công cụ chính: quảng cáo, khuyến mãi, bán hàng cá nhân, quan hệ công chúng (PR), marketing trực tiếp. Trong đó, quảng cáo được hiểu là mọi hình thức giới thiệu và quảng bá phi cá nhân về ý tưởng, sản phẩm, dịch vụ do một nhà tài trợ xác định chi trả (bao gồm quảng cáo trên truyền hình, trên ấn phẩm được in ấn, trên internet, quảng cáo ngoài trời...). Theo định nghĩa trong Pháp lệnh của Ủy ban Thường vụ Quốc hội số 39/2001/PL-UBTVQH10 (16/11/2001), quảng cáo là giới thiệu đến người tiêu dùng về hoạt động kinh doanh, hàng hóa, dịch vụ, bao gồm dịch vụ có mục đích sinh lời và dịch vụ không có mục đích sinh lời.

Từ điển Oxford định nghĩa smartphone là một điện thoại di động thực hiện nhiều chức năng của một máy tính, thường có màn hình giao diện cảm ứng, truy cập internet, và có hệ điều hành có thể chạy các ứng dụng tải về được. Hiệp hội Mobile Marketing toàn cầu định nghĩa: "Mobile marketing là việc sử dụng smartphone như một kênh thông tin, truyền thông, giải trí kết nối giữa doanh nghiệp và người tiêu dùng. Mobile marketing là một kênh Marketing có tính chất tự động, trực tiếp, tương tác, hướng đối tượng vào bất cứ lúc nào, bất kỳ đâu".

Theo Hiệp hội Mobile Marketing, quảng cáo qua smartphone có thể phân chia thành 4 hình thức chính: (1) quảng cáo qua dịch vụ tin nhắn ngắn (Short Messaging Service- SMS)/ dịch vụ tin nhắn đa phương tiện (Multimedia Messaging Service- MMS), (2) quảng cáo trên website điện thoại (mobile web), (3) quảng cáo qua các ứng dụng thông minh (mobile applications), và (4) quảng cáo video (TVC). Một cách khác để quảng cáo thông qua các thiết bị thông minh với việc sử dụng mã phản hồi nhanh (Quick Response CodeQR code) Tuy nhiên, đây là một kỹ thuật đòi hỏi người tiêu dùng phải hành động khi quan tâm trong một quảng cáo.

\section{B. Thái độ đối với quảng cáo qua smartphone}

Theo Kotler và Amstrong [3], thái độ là sự đánh giá tốt xấu, những cảm nghĩ và xu hướng hành động của một cá nhân đối với một đối tượng hoặc một ý tưởng nào đó. Thái độ trong mỗi cá nhân hình thành nên một khung suy nghĩ rằng thích hay không thích một đối tượng đồng thời chấp nhận hay xa lánh, rời bỏ đối tượng đó. Thái độ làm con người có cách hành xử phù hợp với những đối tượng tương tự nhau. Bởi vì thái độ tồn tại ở dạng thức suy nghĩ nên rất khó thay đổi, để thay đổi một thành phần thái độ cụ thể nào đó có thể phải đòi hỏi sự tác động đến nhiều thành phần khác của thái độ.

Thái độ đối với quảng cáo là một khái niệm cốt lõi và cơ bản của một trong những yếu tố để xác định thái độ đối với bất kỳ một quảng cáo cụ thể nào [4]. Thái độ của một người tiêu dùng hay một cá nhân nào đó đối với bất kỳ một hình thức quảng cáo nào cũng bị ảnh hưởng bởi thái độ đối với quảng cáo chung [4].

Nền tảng mô hình nghiên cứu thái độ đối với quảng cáo qua smartphone dựa vào mô hình nghiên cứu thái độ của người tiêu dùng đối với quảng cáo Internet [5], gồm bốn nhân tố ảnh hưởng: (1) giải trí (Entertainment), (2) thông tin (Informativeness), (3) sự làm phiền (Irritation), (4) sự tin tưởng (Credibility). Sau đó, Brackett \& Carr [6] đã sử dụng mô hình và bổ sung thêm các yếu tố nhân khẩu học. Mô hình này được sử dụng phổ biến tại một số nước cho Marketing trên thiết bị thông minh và quảng cáo trực tuyến như Đài Loan [7], Bangladesh [8].

Brackett và Carr [6] chỉ ra rằng thái độ của người tiêu dùng đối với các chương trình quảng cáo trên website bị ảnh hưởng bởi hai nhóm nhân tố chính là: (1) giá trị mà chương trình quảng cáo trên website mang lại và (2) các đặc điểm nhân khẩu học của người tiêu dùng. Trong đó, giá trị của chương trình quảng cáo chịu sự chi phối của bốn nhân tố nhỏ hơn đó là: (1) tính giải trí của chương trình quảng cáo, (2) thông tin và giá trị của thông tin mà chương trình quảng cáo chuyển tải, (3) sự phiền nhiễu mà chương trình quảng cáo gây ra cho người sử dụng website và (4) niềm tin của người tiêu dùng đối với thương hiệu và chương trình được quảng cáo. Tsang và cộng sự [7] bổ sung hai nhân tố (5) sự cho phép 
của người dùng và (6) giá trị khuyến mãi. Xu và cộng sự [9] nghiên cứu tác động của yếu tố tính cá nhân hoá đến thái độ của khách hàng đối với quảng cáo qua smartphone ở Trung Quốc thêm vào (7) tính cá nhân hoá.

Mô hình nghiên cứu thái độ của khách hàng đối với quảng cáo qua tin nhắn văn bản SMS trên smartphone của Trần Thị Thanh Vân [10] dựa trên mô hình của Ducoffe [5] và Tsang và cộng sự [7]), gồm các yếu tố: (1) tính giải trí, (2) giá trị thông tin, (3) sự phiền nhiễu và (4) sự tin tưởng. Mô hình các nhân tố ảnh hưởng đến thái độ của người tiêu dùng trẻ đối với quảng cáo SMS của Phạm Thị Lan Hương và Trần Nguyễn Phương Minh [11] gồm các yếu tố: (1) lợi ích cảm nhận, (2) sự hứng thú cảm nhận, (3) sự tin tưởng, (4) sự cho phép và kiểm soát và (5) sự làm phiền cảm nhận. Nghiên cứu đánh giá tác động của mobile marketing lên thái độ và hành vi khi tiếp nhận quảng cáo của người dùng thông minh tại Thành phố Hồ Chí Minh của Hoàng Hữu Lợi [12] cho thấy các nhân tố tác động: (1) giá trị thông tin, (2) giá trị khuyến mãi, (3) giá trị tin cậy, (4) sự phiền nhiễu, (5) giá trị giải trí và (6) giá trị xã hội.

\section{Mô hình nghiên cứu đề xuất}

Kế thừa Tsang và cộng sự [7] cùng với các nghiên cứu trước, mô hình nghiên cứu được đề xuất như Hình 1.

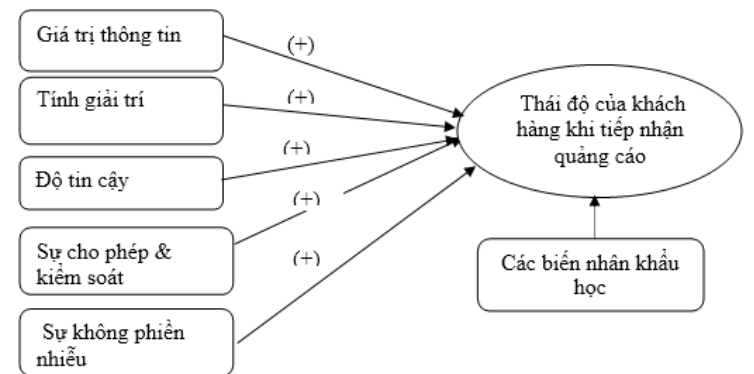

Hình 1: Mô hình nghiên cứu đề xuất (Nguồn: Đề xuất của nhóm tác giả)

Giả thuyết $\mathrm{H}_{1}$ : "Giá trị thông tin của quảng cáo qua điện thoại có tác động cùng chiều đến thái độ của người tiêu dùng đối với quảng cáo qua điện thoại".

Giả thuyết $\mathrm{H}_{2}$ : "Tính giải trí của quảng cáo qua điện thoại có tác động cùng chiều đến thái độ của người tiêu dùng đối với quảng cáo qua điện thoại”.

Giả thuyết $\mathrm{H}_{3}$ : "Độ tin cậy của quảng cáo qua điện thoại có tác động cùng chiều đến thái độ của người tiêu dùng đối với quảng cáo qua điện thoại”.

Giả thuyết $\mathrm{H}_{4}$ : "Sự cho phép và kiểm soát được của quảng cáo qua điện thoại có tác động cùng chiều đến thái độ của người tiêu dùng đối với quảng cáo qua điện thoại".

Giả thuyết $\mathrm{H}_{5}$ : "Sự không phiền nhiễu của quảng cáo qua điện thoại có tác động cùng chiều đến thái độ của người tiêu dùng đối với quảng cáo qua điện thoại”.

\section{KÊT QUẢ NGHIÊN CÚU}

\section{A. Đặc điểm mẫu khảo sát}

Nghiên cứu sử dụng hình thức khảo sát trực tuyến qua e-mail và facebook theo cách chọn mẫu thuận tiện, đối tượng khảo sát có độ tuổi từ $18-35$, là sinh viên, nhân viên văn phòng ở khu vực nội thành của Thành phố Hồ Chí Minh. Họ là những người đã từng và đang sử dụng điện thoại thông minh và thường xuyên sử dụng $3 \mathrm{G}$, wifi để truy cập internet, các ứng dụng điện thoại khác. Chúng tôi thu nhận được 314 phiếu khảo sát, trong đó, có 290 phiếu hợp lệ được dùng để xử lý. Đặc điểm mẫu như trong Bảng 1.

\section{B. Đánh giá độ tin cậy của thang đo}

Kết quả đánh giá các thang đo bằng Cronbach's Alpha (Bảng 2) cho thấy các thang đo đều đạt độ tin cậy Cronbach's Alpha $>0,6$ và hệ số tương quan biến - tổng $>0,3$, tất cả các biến quan sát của các thang đo đều thỏa mãn điều kiện để phân tích EFA.

\section{Phân tích nhân tố khám phá EFA}

Phân tích nhân tố được thực hiện với phép trích Principle Component, phép xoay Varimax cho 22 biến quan sát. Phân tích nhân tố cho thấy hệ số $\mathrm{KMO}=0,897$ đạt yêu cầu $\geq 0,5$; mức ý nghĩa của kiểm định Bartlett $=0,000 \leq 0,05$; tổng phương sai trích được là $65,707 \%$; hệ số tải nhân tố đều $>0,5$ nên đạt yêu cầu. Thang đo chính thức sau khi xử lý EFA gồm 21 biến quan sát (bỏ $\mathrm{NIRR}_{5}$ ) như trong Bảng 3. 
Bảng 1. Thông tin mẫu khảo sát

\begin{tabular}{|c|c|c|c|}
\hline \multicolumn{2}{|c|}{ Thông tin mẫu } & Số lưọng & Tỷ lệ \% \\
\hline \multirow{2}{*}{ Giới tính } & Nam & 126 & 43,4 \\
\hline & Nũ & 164 & 56,6 \\
\hline \multicolumn{2}{|c|}{ Tổng } & 290 & 100 \\
\hline \multirow[t]{2}{*}{ Tuổi } & $\begin{array}{l}18 \text { tuổi }-21 \\
\text { tuổi }\end{array}$ & 151 & 52,1 \\
\hline & $\begin{array}{l}22 \text { tuổi }-35 \\
\text { tuổi }\end{array}$ & 139 & 47,9 \\
\hline \multicolumn{2}{|c|}{ Tổng } & 290 & 100 \\
\hline \multirow[t]{3}{*}{ Trình độ học vấn } & $\begin{array}{l}\text { Từ bậc Trung } \\
\text { học phổ thông } \\
\text { đến Trung cấp }\end{array}$ & 66 & 22,8 \\
\hline & $\begin{array}{l}\text { Cao đẳng, đại } \\
\text { học }\end{array}$ & 154 & 53,1 \\
\hline & Sau đại học & 70 & 24,1 \\
\hline \multicolumn{2}{|c|}{ Tổng } & 290 & 100 \\
\hline \multirow{4}{*}{ Thu nhập } & Dưới 5 triệu & 14 & 4,8 \\
\hline & $\begin{array}{l}5 \text { triệu - dưới } \\
10 \text { triệu }\end{array}$ & 145 & 50,0 \\
\hline & $\begin{array}{l}10 \text { triệu - dưới } \\
20 \text { triệu }\end{array}$ & 91 & 31,4 \\
\hline & Trên 20 triệu & 40 & 13,8 \\
\hline \multicolumn{2}{|c|}{ Tổng } & 290 & 100 \\
\hline
\end{tabular}

(Nguồn: Tính toán của nhóm tác giả)

Bảng 2. Kết quả Cronbach's Alpha các thang đo

\begin{tabular}{|c|c|c|c|c|c|}
\hline STT & Thang đo & $\begin{array}{c}\text { Ký } \\
\text { hiệu }\end{array}$ & $\begin{array}{c}\text { Số } \\
\text { biến } \\
\text { quan } \\
\text { sát }\end{array}$ & $\begin{array}{l}\text { Hệ số } \\
\text { Cron- } \\
\text { bach's } \\
\text { Al- } \\
\text { pha }\end{array}$ & $\begin{array}{l}\text { Hệ số } \\
\text { tương } \\
\text { quan } \\
\text { biến- } \\
\text { tô̂ng } \\
\text { nhỏ } \\
\text { nhất }\end{array}$ \\
\hline 1 & $\begin{array}{c}\text { Giá trị thông } \\
\text { tin }\end{array}$ & INF & 4 & 0,804 & 0,498 \\
\hline 2 & Tính giải trí & ENT & 5 & 0,871 & 0,643 \\
\hline 3 & $\begin{array}{c}\text { Sự không } \\
\text { phiền nhiễu }\end{array}$ & NIRR & 5 & 0,864 & 0,647 \\
\hline 4 & Độ tin cậy & CRE & 4 & 0,798 & 0,509 \\
\hline 5 & $\begin{array}{l}\text { Sự cho phép } \\
\text { và kiểm soát }\end{array}$ & PRC & 4 & 0,787 & 0,513 \\
\hline 6 & $\begin{array}{c}\text { Thái độ đối } \\
\text { với quảng } \\
\text { cáo } \\
\text { smartphone }\end{array}$ & ATT & 2 & 0,823 & 0,708 \\
\hline
\end{tabular}

(Nguồn: Tính toán của nhóm tác giả)
Bảng 3. Kết quả ma trận xoay nhân tố

\begin{tabular}{|l|c|c|c|c|c|}
\hline \multirow{2}{*}{} & \multicolumn{5}{|c|}{ Nhân tố } \\
\hline & 1 & 2 & 3 & 4 & 5 \\
\hline $\mathrm{NIRR}_{3}$ & 0,814 & & & & \\
\hline $\mathrm{NIRR}_{2}$ & 0,759 & & & & \\
\hline $\mathrm{NIRR}_{1}$ & 0,747 & & & & \\
\hline $\mathrm{NIRR}_{4}$ & 0,716 & & & & \\
\hline $\mathrm{ENT}_{3}$ & & 0,801 & & & \\
\hline $\mathrm{ENT}_{2}$ & & 0,770 & & & \\
\hline $\mathrm{ENT}_{4}$ & & 0,732 & & & \\
\hline $\mathrm{ENT}_{1}$ & & 0,699 & & & \\
\hline $\mathrm{ENT}_{5}$ & & 0,637 & & & \\
\hline $\mathrm{CRE}_{2}$ & & & 0,783 & & \\
\hline $\mathrm{CRE}_{3}$ & & & 0,766 & & \\
\hline $\mathrm{CRE}_{1}$ & & & 0,745 & & \\
\hline $\mathrm{CRE}_{4}$ & & & 0,606 & & \\
\hline $\mathrm{PRC}_{2}$ & & & & 0,881 & \\
\hline $\mathrm{PRC}_{4}$ & & & & 0,782 & \\
\hline $\mathrm{PRC}_{1}$ & & & & 0,732 & \\
\hline $\mathrm{PRC}_{3}$ & & & & 0,727 & \\
\hline $\mathrm{INF}_{4}$ & & & & & 0,759 \\
\hline $\mathrm{INF}_{1}$ & & & & & 0,647 \\
\hline $\mathrm{INF}_{2}$ & & & & & 0,646 \\
\hline $\mathrm{INF}_{3}$ & & & & & 0,644 \\
\hline & & & & & \\
\hline
\end{tabular}

(Nguồn: Tính toán của nhóm tác giả)

Phân tích nhân tố biến phụ thuộc cho kết quả phương sai trích $65,707 \%$ ở lần phân tích nhân tố đầu tiên, và các biến đều có hệ số tải nhân tố đều lớn hơn 0,5 và mức sig. của Bartlett là 0,000 $<0,05$. Như vậy, cả hai biến quan sát của biến thái độ được tiếp tục đưa vào những phân tích tiếp theo.

\section{Mô hình hồi quy tuyến tính}

Kết quả trong phân tích ma trận tương quan Pearson đã chỉ ra rằng các hệ số tương quan đều có ý nghĩa thống kê (Sig. < 0,01). Vì vậy, tất cả các biến sẽ được sử dụng trong phân tích hồi quy ở bước tiếp theo.

Bảng 4 cho thấy $\mathrm{R}^{2}$ hiệu chỉnh bằng $51 \%$, tức là mô hình này với năm biến độc lập giải thích được $51 \%$ biến thiên của thái độ đối với quảng cáo qua smartphone và $49 \%$ còn lại là do các biến khác ngoài mô hình mà nghiên cứu này chưa đề cập đến.

Bảng 5 cho thấy giá trị sig. của mô hình rất 
Bảng 4. Kết quả tóm tắt mô hình hồi quy tuyến tính

\begin{tabular}{|c|c|c|c|c|}
\hline Hệ số R & $\begin{array}{c}\text { Hệ số } \\
\text { xác định } \\
-\mathrm{R}^{2}\end{array}$ & $\begin{array}{l}\text { Hệ số R } \\
\text { hiệu chỉnh }\end{array}$ & $\begin{array}{c}\text { Sai số } \\
\text { chuẩn của } \\
\text { ước lượng }\end{array}$ & $\begin{array}{l}\text { Chỉ số } \\
\text { Durbin- } \\
\text { Watson }\end{array}$ \\
\hline 0,720 & 0,519 & 0,510 & 0,63262 & 1,448 \\
\hline \multicolumn{5}{|c|}{ Biến độc lập: (Constant), INF, ENT, NIRR, CRE, PRC } \\
\hline \multicolumn{5}{|c|}{$\begin{array}{l}\text { Biến phụ thuộc: Thái độ đối với quảng cáo qua điện } \\
\text { thoại - ATT }\end{array}$} \\
\hline
\end{tabular}

(Nguồn: Tính toán của nhóm tác giả)

Bảng 5. Kết quả kiểm định mô hình hồi quy hồi quy tuyến tính bội

\begin{tabular}{|c|l|c|c|c|c|c|}
\hline \multicolumn{2}{|c|}{} & $\begin{array}{c}\text { Tổng } \\
\text { bình } \\
\text { phương }\end{array}$ & $\begin{array}{c}\text { Bậc } \\
\text { tự } \\
\text { do }\end{array}$ & $\begin{array}{c}\text { TB } \\
\text { Bình } \\
\text { phương }\end{array}$ & $\begin{array}{c}\text { Hệ } \\
\text { số } \\
\mathrm{F}\end{array}$ & $\begin{array}{c}\text { Giá } \\
\text { trị } \\
\text { Sig. }\end{array}$ \\
\hline \multirow{3}{*}{1} & Hồi quy & 122,497 & 5 & 24,499 & 61,216 & $0,000 \mathrm{~b}$ \\
\cline { 2 - 7 } & Phần dư & 113,659 & 284 & 0,400 & & \\
\cline { 2 - 7 } & Tồng & 236,156 & 289 & & & \\
\hline
\end{tabular}

(Nguồn: Tính toán của nhóm tác giả)

nhỏ so với mức ý nghĩa (sig. $=0,000<0,05$ ), như vậy mô hình phù hợp với tập dữ liệu và có thể suy rộng ra cho tổng thể. Bảng 6 cho thấy giá trị sig. của các biến độc lập đều nhỏ hơn mức ý nghĩa 0,05 , nghĩa là các biến độc lập tương quan cao, ảnh hưởng đáng kể đến biến phụ thuộc. Các hệ số hồi quy Beta chuẩn hóa đều có giá trị dương tức là chúng tác động cùng chiều đối với biến phụ thuộc. Như vậy, mô hình phù hợp với các giả thuyết ban đầu, các giả thuyết $\mathrm{H}_{1}, \mathrm{H}_{2}, \mathrm{H}_{3}, \mathrm{H}_{4}, \mathrm{H}_{5}$ được chấp nhận. Mức độ tác động giảm dần như sau: (1) tính giải trí, (2) giá trị thông tin, (3) độ tin cậy, (4) sự cho phép và kiểm soát và (5) sự không phiền nhiễu. Như vậy, mô hình hồi quy bội lúc này sẽ được viết lại như sau: Thái độ đối với quảng cáo $=-0,467$ $+0,240^{*}$ Giá trị thông tin $+0,351^{*}$ Tính giải trí $+0,166^{*}$ Sự không phiền nhiê̂u $+0,203^{*}$ Độ tin cậy $+0,137 *$ Sự cho phép và kiểm soát $+\mathrm{e}_{i}$

Trong việc dò tìm sự vi phạm các giả định hồi quy tuyến tính: biểu đồ phân tán Scatterplot cho thấy phần dư không thay đổi theo một trật tự nào đối với giá trị dự đoán, chúng phân tán ngẫu nhiên, giả thuyết về liên hệ tuyến tính không bị vi phạm. Hệ số tương quan hạng Spearman của giá trị tuyệt đối phần dư và các biến độc lập: giá trị Sig. của các hệ số tương quan với độ tin cậy $95 \%$ đều lớn hơn 0,05 , cho thấy phương sai của sai số không thay đổi, giả định không bị vi phạm. Biểu đồ Histogram cho thấy phần dư có phân phối chuẩn với giá trị trung bình rất nhỏ gần bằng $0(\mathrm{Mean}=1,43 \mathrm{E}-15)$ và độ lệch chuẩn của nó gần bằng $1(0,989)$, đồ thị P-P plot biểu diễn các điểm quan sát thực tế tập trung khá sát đường chéo những giá trị kỳ vọng, có nghĩa là dữ liệu phần dư có phân phối chuẩn. Hệ số $1<$ Durbin -Watson $=1,448<3$ là thỏa điều kiện, hệ số phóng đại phương sai VIF $<10$ cho thấy các biến độc lập không có quan hệ chặt chẽ với nhau nên không xảy ra hiện tượng đa cộng tuyến. Như vậy, mô hình hồi quy tuyến tính được xây dựng theo phương trình trên không vi phạm các giả định cần thiết trong hồi quy tuyến tính.

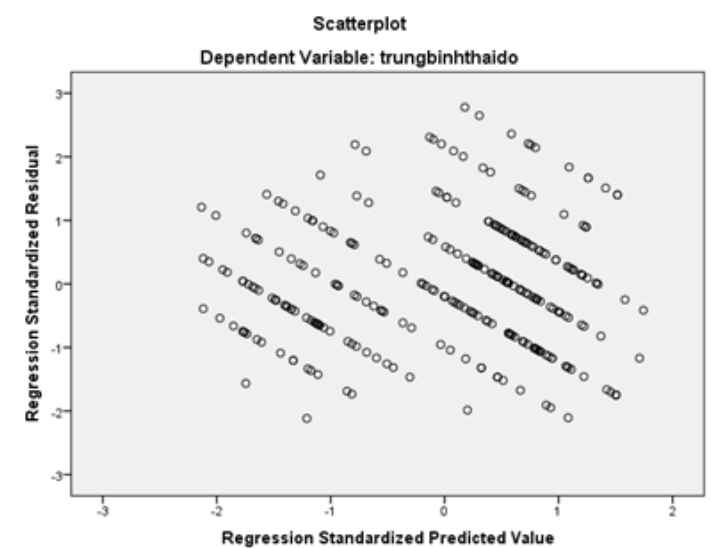

Hình 2: Biểu đồ phân tán Scatterplot (Nguồn: Kết quả phân tích SPSS)

\section{Kiểm định sụ̣ khác biệt}

Về giới tính, kết quả kiểm định Independentsamples T-test cho thấy kiểm định Levene Test đã được tiến hành trước với kết quả sig. bằng $0,09>0,05$ cho thấy phương sai của trung bình thái độ đối với quảng cáo có phân phối chuẩn nên sig. của kiểm định $\mathrm{t}$ được tính là $0,184>$ 0,05, không có sự khác biệt về thái độ đối với quảng cáo qua smartphone giữa nam và nữ tại Thành phố Hồ Chí Minh.

Về tuổi, kiểm định Levene test có kết quả với hệ số sig. $0,844>0,05$, tức là phương sai này có phân phối chuẩn. Kết quả phân tích ANOVA sig. $=0,795>0,05$, không có sự khác biệt giữa các nhóm tuổi đối với thái độ quảng cáo qua smartphone. 
Bảng 6. Kết quả kiểm định thống kê các hệ số hồi quy

\begin{tabular}{|c|c|c|c|c|c|c|c|c|}
\hline & \multicolumn{2}{|c|}{$\begin{array}{l}\text { Hệ số chưa, } \\
\text { chuẩn hóa }\end{array}$} & \multirow{2}{*}{$\begin{array}{c}\begin{array}{c}\text { Hệ số, } \\
\text { chuẩn hóa }\end{array} \\
\text { Beta }\end{array}$} & \multirow{2}{*}{$\begin{array}{c}\text { Kiểm định } \\
\mathrm{t}\end{array}$} & \multirow{2}{*}{$\begin{array}{c}\text { Sig., } \\
\text { (Mức ý nghĩa) }\end{array}$} & \multicolumn{2}{|c|}{$\begin{array}{l}\text { Thống kê đa, } \\
\text { cộng tuyến }\end{array}$} \\
\hline & & B & $\begin{array}{c}\text { Sai } \\
\text { số chuẩn }\end{array}$ & & & & $\begin{array}{c}\text { Độ } \\
\text { chấp nhận }\end{array}$ & VIF \\
\hline \multirow{6}{*}{1} & Hằng số & $-0,467$ & 0,252 & & $-1,853$ & 0,065 & & \\
\hline & CRR & 0,240 & 0,061 & 0,218 & 3,938 & 0,000 & 0,554 & 1,805 \\
\hline & SR & 0,351 & 0,062 & 0,323 & 5,644 & 0,000 & 0,517 & 1,933 \\
\hline & EO & 0,166 & 0,060 & 0,151 & 2,745 & 0,006 & 0,556 & 1,797 \\
\hline & SQ & 0,203 & 0,058 & 0,180 & 3,518 & 0,001 & 0,648 & 1,542 \\
\hline & CR & 0,137 & 0,048 & 0,119 & 2,878 & 0,004 & 0,993 & 1,007 \\
\hline
\end{tabular}

(Nguồn: Tính toán của nhóm tác giả)

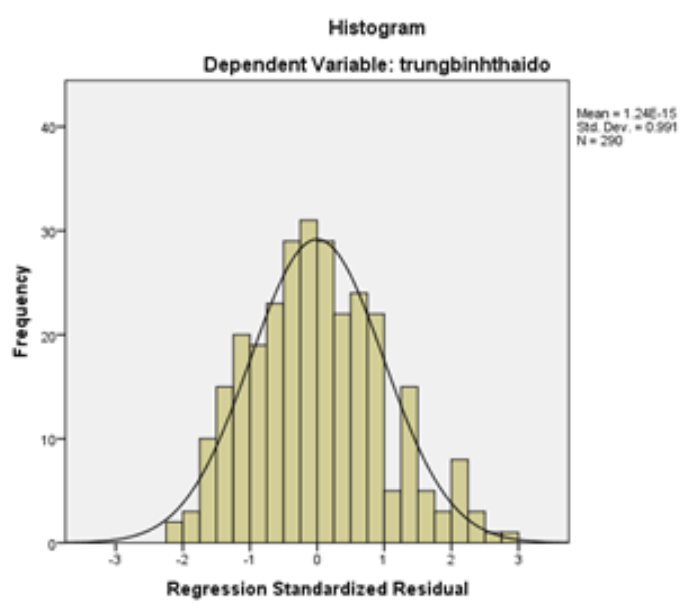

Hình 3: Biểu đồ Histogram (Nguồn: Kết quả phân tich SPSS)

Về trình độ học vấn, chỉ số sig. của Levene test là $0,01<0,05$ điều này có nghĩa là phương sai các nhóm không có phân phối chuẩn hay phương sai các nhóm khác nhau, ngoài ra hệ số sig. ANOVA là $0,002<0,05$, trình độ học vấn có sự khác biệt với thái độ quảng cáo qua điện thoại. Kiểm định Tamhane's T2 cho thấy có sự khác biệt rõ rệt giữa các nhóm có trình độ học vấn khác nhau.

Về thu nhập, hệ số sig. $0,072>0,05$ tức là phương sai này có phân phối chuẩn. Kết quả phân tích ANOVA sig. $=0,00<0,05$, có sự khác biệt giữa các nhóm thu nhập đối với thái độ khi xem quảng cáo qua điện thoại. Kiểm định Dunnet cho thấy giữa ba nhóm có thu nhập khác nhau có sự khác biệt đối với thái độ quảng cáo qua điện thoại.

\section{KÊTT LUẬN VÀ HÀM Ý QUẢN TRI}

\section{A. Kết luận}

Nghiên cứu sử dụng phương pháp lấy mẫu thuận tiện, 290 phiếu đạt yêu cầu phân tích và xử lý dữ liệu. Sau khi đánh giá độ tin cậy bằng Cronbach's alpha, và phân tích nhân tố khám phá EFA, rút ra được 5 nhân tố như mô hình đề xuất. Kết quả mô hình hồi quy tuyến tính có $\mathrm{R}^{2}$ hiệu chỉnh là $51 \%$. Các biến độc lập đều có tác động cùng chiều đến thái độ của người tiêu dùng đối với quảng cáo qua smartphone tại Thành phố Hồ Chí Minh. Kiểm định cho thấy có sự khác biệt về thái độ đối với quảng cáo qua smartphone tại Thành phố Hồ Chí Minh theo hai đặc điểm cá nhân: thu nhập bình quân, trình độ học vấn, trong khi hai đặc điểm còn lại: giới tính, độ tuổi không cho thấy sự khác biệt. Mức độ tác động giảm dần như sau: (1) tính giải trí, (2) giá trị thông tin, (3) độ tin cậy, (4) sự cho phép và kiểm soát và (5) sự không phiền nhiễu.

\section{B. Hàm ý quản trị}

- Đối với Tính giải trí

Đây là yếu tố có ảnh hưởng mạnh nhất (beta $=$ $0,323)$, và mức ý nghĩa đang có chiều hướng tích cực với mean $=3,358$. Những nội dung quảng cáo mang tính giải trí cao, cách thể hiện truyền đạt nội dung quảng cáo mang tính hài hước, gần gũi sẽ dễ dàng đi vào tâm thức khách hàng. Các yếu tố ảnh hưởng đến tính giải trí bao gồm: thú vị, thoải mái, thư giãn, giải trí, bắt mắt, hài lòng.

Quảng cáo bằng hình ảnh qua điện thoại hiện nay chủ yếu là quảng cáo bằng hình thức banner. 
Với giao diện bị hạn chế khá nhiều, kích thước nhỏ, do đó, các nhà marketing nên quan tâm đầu tư hơn về chất lượng, giá trị, nội dung phải mang tính giải trí, hài hước, thư giãn. Người tiêu dùng càng ngày càng thông minh và khó tính, vì vậy chỉ những quảng cáo có ý tưởng mới, độc đáo hoặc đáp ứng đúng nhu cầu của khách hàng thì mới có thể thu hút được sự chú ý của người xem. Nếu cần thiết, có thể kết hợp với âm thanh, hình ảnh để tạo nên sự sống động cho quảng cáo và đặc biệt là chạm đến cảm xúc của người xem thì mới có thể gây được sự chú ý của khách hàng.

- Đối với Giá trị thông tin

Yếu tố này có mức ảnh hưởng thứ hai, mức ý nghĩa trung bình $($ mean $=3,16)$. Có thể nhận định rằng thông tin quảng cáo qua smartphone chưa đầy đủ so với các quảng cáo trên trang web của doanh nghiệp. Chỉ khi cần, người tiêu dùng mới chủ động tìm đến trang web doanh nghiệp, còn quảng cáo qua smartphone có thể họ chưa cần, nhưng thông qua các quảng cáo này họ có cơ hội được biết đến thương hiệu, sản phẩm, dịch vụ và tạo ra hay kích thích nhu cầu cho họ. Vì vậy, các doanh nghiệp nên cung cấp những thông tin sản phẩm, dịch vụ một cách thường xuyên và liên tục để thu hút sự quan tâm, chú ý của người tiêu dùng. Chính vì thế, đối với quảng cáo qua smartphone, ngoài tính hài hước, giải trí, các nhà quản trị nên tập trung về cách diê̂n đạt, trình bày thông tin một cách ngắn gọn, gần gũi và dễ hiểu nhất sẽ giúp ghi nhớ hơn trong tâm trí khách hàng.

- Đối với Độ tin cậy

Yếu tố này có ảnh hưởng thứ ba, với mức ý nghĩa tích cực mean $=3,24$. Người tiêu dùng cho rằng môi trường liên quan đến internet là môi trường tự do ngôn luận, chính vì vậy tính xác thực cũng như độ tin cậy của các thông tin quảng cáo không được đảm bảo, làm cho người tiêu dùng bị nhiễu. Sự tin cậy của người tiêu dùng có được từ quảng cáo smartphone có thể là từ kinh nghiệm của bản thân hoặc được sự giới thiệu của người thân và bạn bè. Doanh nghiệp cần chú ý đến cách tiếp cận trực tiếp với người tiêu dùng thông qua smartphone để tạo sự gần gũi, thân thiện, thông hiểu giữa doanh nghiệp và người tiêu dùng, mang lại giá trị cho người tiêu dùng, từ đó chiếm được lòng tin của họ.
- Đối với Sự không phiền nhiễu

Yếu tố có mức ảnh hưởng đứng thứ tư, nhưng có giá trị trung bình thấp nhất mean $=3,11$, người tiêu dùng có thái độ chưa thực sự tốt đối với yếu tố này. Người tiêu dùng có quyền lựa chọn xem quảng cáo có nghĩa là họ chủ động chứ không rơi vào tình trạng bị động. Đồng thời, các chính sách quảng cáo trên điện thoại cần luôn được điều chỉnh thường xuyên để hạn chế sự làm phiền, ảnh hưởng đến người dùng. Ngoài ra, các kỹ thuật quảng cáo trên điện thoại cần luôn được cải tiến và ngày càng thân thiện với người dùng. Các nhà quản trị nên xây dựng nên những chiến lược quảng cáo thích hợp, tạo cho họ sự thoải mái và không gây phiền nhiễu cho người tiêu dùng.

- Đối với Sự cho phép và kiểm soát

Yếu tố này có mức độ ảnh hưởng thấp nhất, tuy nhiên lại có mức ý nghĩa cao nhất mean = 3,48 . Đối với người tiêu dùng sử dụng điện thoại, việc làm chủ mọi quảng cáo, mọi yếu tố gây phiền nhiễu, được kiểm soát và làm chủ được những quảng cáo với bản thân sẽ giúp họ thấy thỏai mái hơn. Yếu tố "có thể từ chối việc nhận quảng cáo" là yếu tố mức ý nghĩa cao nhất trong 21 biến quan sát của mô hình. Đa số khách hàng cho rằng mỗi ngày họ có quá nhiều quảng cáo qua smartphone. Do đó, các nhà marketing nên nghiên cứu tìm hiểu những hình thức quảng cáo phù hợp, có cho phép người tiêu dùng từ chối hoặc kiểm soát mọi quảng cáo nếu họ không thích. Các doanh nghiệp cũng nên sử dụng các công cụ có thể lọc được đối tượng khách hàng phù hợp, nghiên cứu kỹ hơn về hành vi của khách hàng trên điện thoại.

- Đối với các yếu tố nhân khẩu học

Đối với yếu tố thu nhập, có sự khác biệt về thái độ giữa các nhóm có thu nhập khác nhau. Điều này cũng dễ hiểu, thu nhập cũng là một trong những quyết định hành vi tiêu dùng của khách hàng. Chính vì vậy, khi doanh nghiệp phân khúc thương hiệu/sản phẩm/dịch vụ theo thu nhập thì cần chú ý đến thông điệp truyền tải dành cho từng nhóm cũng như cách thức thể hiện thông điệp đó trong quảng cáo. Sự khác biệt này cũng giúp cho các doanh nghiệp xây dựng các chiến lược quảng cáo khác nhau cho các nhóm thu nhập khác nhau.

Đối với trình độ học vấn, sự khác biệt khá rõ rệt. Ở mỗi trình độ khác nhau, nhận thức của mỗi 
người cũng sẽ khác nhau. Quảng cáo hướng đến đối tượng có trình độ thấp thì cần dùng những từ ngữ dễ hiểu, thông dụng, gần gũi. Ngược lại, những quảng cáo hướng đến đối tượng có trình độ cao thì nội dung phải mang hình ảnh sang trọng, có tính khoa học, nội dung câu chữ phù hợp với người tiếp nhận.

\section{Hạn chế của bài viết}

Nghiên cứu chỉ được thực hiện trong khu vực Thành phố Hồ Chí Minh, cụ thể tại một số trường đại học và một số nhân viên văn phòng, do đó tính đại diện còn hạn chế. Nghiên cứu chọn mẫu phi xác suất và cách lấy mẫu thuận tiện, làm cho tính đại diện không cao. Nghiên cứu có thể vẫn chưa bao gồm các khía cạnh khác ảnh hưởng đến thái độ của người tiêu dùng đối với quảng cáo qua smartphone như tính tương tác, tính cá nhân hóa, quyết định mua hàng... Đó cũng chính là những gợi ý cho những nghiên cứu về sau.

\section{TÀI LIẸU THAM KHẢO}

[1] Bộ Thông tin và Truyền thông. Báo cáo so kết 06 tháng đầu năm; 2015.

[2] MMA. Mobile Marketing Association; 2008.

[3] Kotler P, Amstrong G. Quản trị marketing. Thành phố Hồ Chí Minh: Nhà Xuất bản Lao động - Xã hội; 2013.

[4] Lutz Richard J. Affective and Cognitive Antecedents of Attitude Toward the Ad: A Conceptual Framework, in Psychological Processes and Advertising Effectsn. Alvitt LF, Mitchell AA, editors. Hillsdale, NJ: Lawrence Erlbaum Associates; 1985.

[5] Ducoffe R H. Advertising Value and Advertising on the Web. Journal of Advertising Research. 1996;36(5):21-35.

[6] Brackett L K, Carr B N. Cyberspace Advertising vs. Other Media: Consumer vs. Mature Student Attitudes. Journal of Advertising Research. 2001;41(5):23-32.

[7] Tsang M M, Ho S C, Liang T P. Consumer Attitudes Toward Mobile Advertising: An Empirical Study. International Joural of Electronic Commerce. 2004;8:65-78.

[8] Chowdhury $\mathrm{H} \mathrm{K}$, Parvin N, Weitenberner C, Becker M. Consumer attitude toward mobile advertising in an emerging market: An empirical study. Marketing. 2010;12(2):206-216.

[9] Xu D J, Liao S S, Li Q. Combining empirical experimentation and modeling techniques: A design research approach for personalized mobile advertising applications. Decision Support Systems. 2008;44:710-724.
[10] Trần Thị Thanh Vân. Các yếu tố tác động đến thái độ của khách hàng tại TPHCM đối với quảng cáo qua tin nhắn văn bản SMS trên smartphone [Luận văn Thạc sĩ]; 2013.

[11] Phạm Thị Lan Hương, Trần Nguyễn Phương Minh. Các nhân tố ảnh hưởng đến thái độ của người tiêu dùng trẻ đối với quảng cáo SMS. Tạp chi Phát triển Kinh tế. 2014;286:89-108.

[12] Hoàng Hữu Lợi. Nghiên cứu đánh giá tác động của Mobile Marketing lên thái độ của hành vi khi tiếp nhận quảng cáo của người dùng thông minh tại Thành phố Hồ Chí Minh [Luận văn Thạc sĩ]; 2013. 